\title{
The onset of molecular condensation: hydrogen
}

\author{
J. H. Morilla, J. M. Fernández, G. Tejeda and S. Montero* \\ Received 18th February 2010, Accepted 18th May 2010 \\ DOI: $10.1039 / \mathbf{c 0 0 3 3 7 6 f}$
}

The very first steps of condensation as studied experimentally in the simplest molecular system (para- $\mathrm{H}_{2}$ ) are reported. The fast time-space evolution of the nascent clusters have been measured using state-of-the-art Raman spectroscopy implemented on cryogenic supersonic jets. The time-dependent onset of condensation is presented in a non-equilibrium pressure-temperature phase diagram. Dimer and trimer formation are found to obey three-body processes whose rates have been determined.

\section{Introduction}

An unsolved fundamental problem in condensed matter physics of classical and quantum systems concerns how homogeneous condensation actually starts. ${ }^{1}$ It is known that the formation and evolution of large clusters strongly depends on the preexisting smaller units, in particular on the abundance of dimers. ${ }^{2}$ However, the crucial question of how the trigger units, dimers and trimers, are formed via either two-body or three-body processes and at what rate, has not been answered unambiguously to date. ${ }^{3}$ This precludes a clear understanding of matter growth, in particular in quantum systems like clusters of $\mathrm{He}$ or para- $\mathrm{H}_{2}$ where structure, dynamics, and kinetics are deeply interwoven in the very first steps of homogeneous condensation. Many theoretical works on para- $\mathrm{H}_{2}$ clusters ${ }^{4,5}$ point at these questions, with the added value of a possible new state of matter in the scene: molecular superfluidity, which has been predicted by theory ${ }^{6}$ but only indirect evidence has been found experimentally in mixed clusters. ${ }^{7}$ Superfluidity in pristine $\left(\mathrm{pH}_{2}\right)_{N}$ clusters seems to be constrained to $N \leq 26$ at $T<1.5 \mathrm{~K},{ }^{5}$ and its occurrence might be conditioned by the first steps of clustering considered here.

Hydrogen dimers have been investigated by infrared spectroscopy in bulk ${ }^{8}$ and in jets by diffractive scattering on crystal surfaces, ${ }^{9}$ while larger clusters have been studied in jets by mass spectrometry, ${ }^{10}$ depletion spectroscopy, ${ }^{7}$ Rayleigh-,${ }^{11}$ and Raman scattering. ${ }^{12-14}$ Raman scattering seems the ideal spectroscopic technique to detect neutral $\mathrm{pH}_{2}$ molecules, either free or aggregated in clusters. Its crucial advantage is the non-intrusive observation and accurate measure of abundances of the nascent clusters with size resolution, at the time and spot where they are being formed. ${ }^{12}$ Furthermore, it overcomes the many ambiguities from most experimental methods, which are largely based on terminal molecular beam conditions with mass spectroscopic detection subject to controversial fragmentation/recombination processes. ${ }^{15}$

On the basis of experimental data of unprecedented accuracy we report here a detailed quantitative study of the formation and evolution of small $\left(\mathrm{pH}_{2}\right)_{N}$ clusters focused on the

Laboratory of Molecular Fluid Dynamics@ Instituto de Estructura de la Materia, CSIC Serrano 121, E-28006 Madrid, Spain.

E-mail:emsalvador@iem.cfmac.csic.es kinetics of dimer and trimer formation. The work is aimed at establishing the basis for rigorous quantitative theories of cluster growth by providing a wealth of experimental data on the most elementary condensation processes of the simplest molecular system conceivable: para- $\mathrm{H}_{2}$.

\section{Experimental}

We employ here an upgraded apparatus with respect to our previous works, ${ }^{12,14}$ with a better spectral resolution of up to $0.11 \mathrm{~cm}^{-1}$, a high-accuracy mass-flow control system, and a lowest temperature in the source of $22 \mathrm{~K}$ at 0.18 bars, up to $46 \mathrm{~K}$ at 2 bars stagnation pressure. Source temperatures regulated to $\pm 0.01 \mathrm{~K}$ provide a long term thermal stability in the jet of several hours. These conditions are a must in order to span the existence domain of the small $\mathrm{pH}_{2}$ clusters, to discriminate them by size, and to measure their local abundances along the jet with better accuracy than in our preliminary study. ${ }^{14}$

A scheme of one of the many runs of the experiment is shown in Fig. 1, with the $D=50 \mu \mathrm{m}$ nozzle and the flow field (middle), the actual size and the flow quantities (bottom), and three representative Raman spectra of $\left(\mathrm{pH}_{2}\right)_{N}$ clusters in the region of the Q-branch of the fundamental vibrational band of $\mathrm{pH}_{2}$ as observed at different points of the jet (top). The size of the actual Raman scattering volume $\mathscr{R}$ seen by the detector of the spectrometer is $\approx 90 \times 15 \times 2 \mu \mathrm{m}^{3}$. Residual pressure in the expansion chamber was $P_{r} \approx 0.003$ mbar, the normal shock wave being located far away from the region under study $(1 \leq z / D \leq 26)$, and not interfering with it. The primary quantities of this experiment are the positions $z$ of the volume $\mathscr{R}$ along the jet axis and the number densities $n_{N}(z)$ of $\mathrm{pH}_{2}$ molecules in $\mathscr{R}$, either free $(N=1)$, or aggregated in clusters of size $N .{ }^{16}$

Positions $z$ were set displacing smoothly the nozzle and its flow field along its axis by means of a high resolution micropositioning stage of $\pm 0.1 \mu \mathrm{m}$ precision, maintaining the observation volume $\mathscr{R}$ fixed at the intersection between the sharply focused $c w$-laser beam $(\lambda=514.5 \mathrm{~nm}$, singlemode, $5 \mathrm{~W}$ ) and the optical axis of the spectrometer. Reference position $z=0$ was determined by means of the magnified shadow $(\times 10)$ of the nozzle exit onto the entrance slit of the spectrometer. 


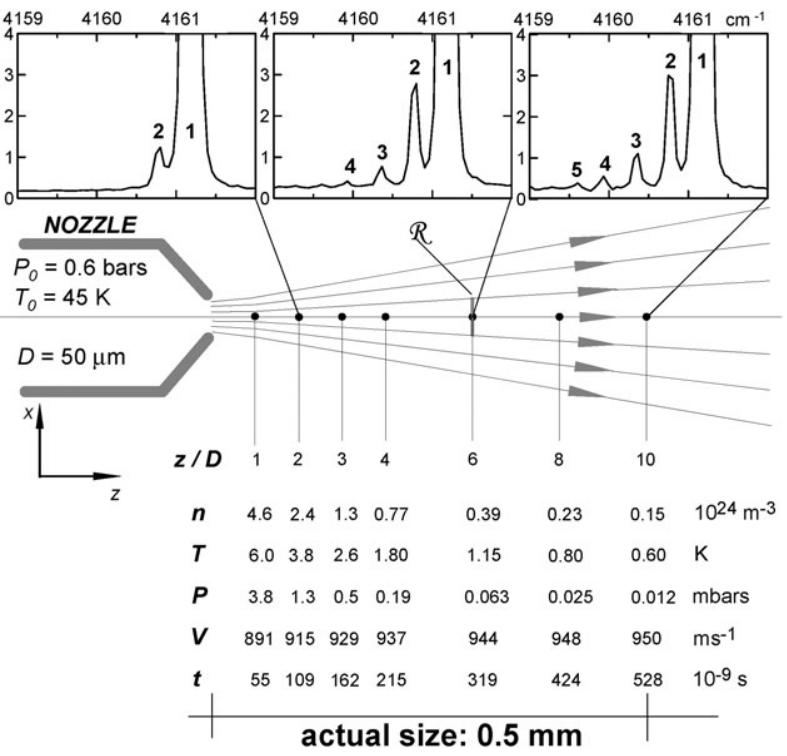

Fig. 1 Supersonic free jet of $\mathrm{pH}_{2}$ (middle), with flow quantities (bottom) and Raman spectra of $\left(\mathrm{pH}_{2}\right)_{N}$ clusters (top). Intensity scale refers to the monomer intensity $I_{1}=100$; number densities $n_{N}(z)$ are strictly proportional to intensities $I_{N}$.

The clusters were identified by their $\Delta \nu$ red-shifts on the basis of the assignment proposed in ref. 12. Since the intermolecular distances within the clusters are typically larger than $4 \AA^{12}$ (negligible electronic interaction), the polarizability of the clusters grows linearly with the number of $\mathrm{pH}_{2}$ units as long as the cluster size remains smaller than the $514.5 \mathrm{~nm}$ excitation wavelength. This allows for an accurate determination of the number densities $n_{N}(z)$, which are strictly proportional to the intensities $I_{N}$. The total number density $n(z)=\sum_{N} n_{N}(z)$ was measured from the integrated Raman intensity of the spectral region $4159 \leq \nu \leq 4162 \mathrm{~cm}^{-1}$ at a number of points along the jet, comparing this intensity with that of the homologous spectrum recorded under static conditions, 14 mbars and $295 \mathrm{~K}$, and identical optical path.

The other flow quantities shown in Fig. 1 were obtained as follows: translational temperatures $T(z)$ were derived from $n(z)$ and stagnation conditions, $n_{0}$ and $T_{0}$, by means of the isentropic relation of a $\gamma=5 / 3$ ideal gas, which is a good approximation for $\mathrm{pH}_{2}$ at the temperatures of the flow field. The contribution of condensation heat to the flow temperature has been neglected due to the small size of the clusters and its low abundance. Pressures $P(z)$ were derived from $n(z)$ and $T(z)$ using the equation of state. The local flow velocities $V(z)=\sqrt{5\left(T_{0}-T(z)\right) R / W}$ were obtained from the conservation of total energy along the jet; $R=8.31451 \mathrm{~J} \mathrm{~K}^{-1} \mathrm{~mol}^{-1}$ is the universal gas constant, and $W=0.002 \mathrm{~kg} \mathrm{~mol}^{-1}$ the molar mass of $\mathrm{H}_{2}$. Eventually, the time $t$ elapsed from the beginning of the expansion was determined from $z$ and $V(z)$.

\subsection{Measurements}

In the first part of this work the influence of the stagnation conditions $P_{0}$ and $T_{0}$ onto the size and abundance of the $N \leq 6$ clusters has been explored systematically along the jet.

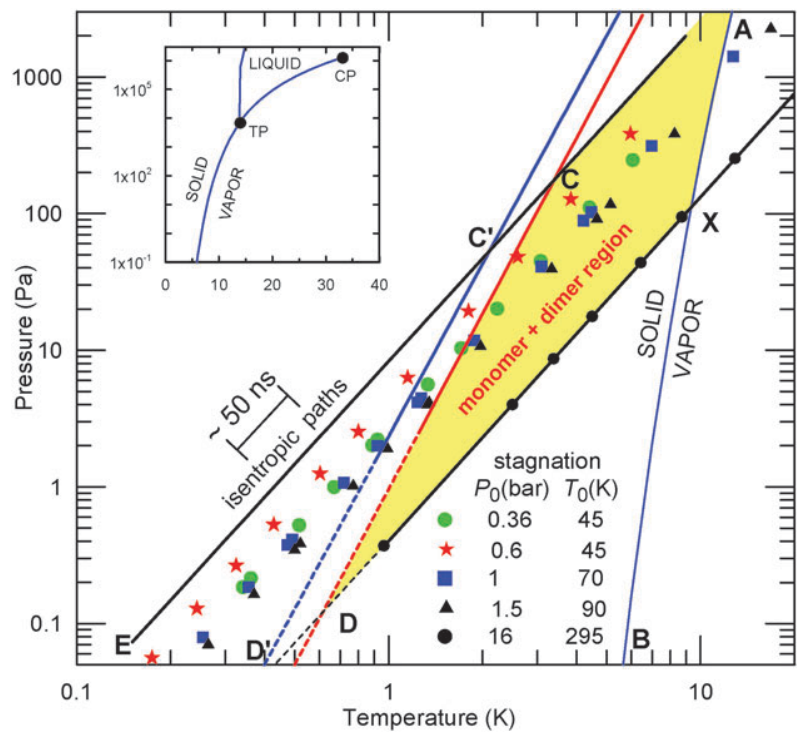

Fig. 2 Isentropic paths in $P T$ phase diagram of $\mathrm{pH}_{2}$ for some of the jets investigated in this work. Symbols stand for the measured points. Boundaries of maximum cluster size regions are shown.

The phase diagram on Fig. 2 (inset) represents the stability regions corresponding to the well defined solid, liquid, and gas phases under equilibrium conditions. According to that diagram, slow, quasi-equilibrium transformations across the $P T$-space do not allow neither formation nor observation of $\left(\mathrm{pH}_{2}\right)_{N}$ clusters: when the gas crosses the saturation line a sudden condensation into solid (below the triple point TP), or liquid (above TP) occurs, with little chance to observe short-life intermediate species.

Supersonic expansions overcome these limitations in two ways. First, by extending the time scale over a large space domain by means of the high flow velocity $V$, which ranges here from $\approx 800$ to $\approx 1000 \mathrm{~m} \mathrm{~s}^{-1}$ (see Fig. 1); for such velocities, $1 \mu$ s corresponds to $1 \mathrm{~mm}$, which is the overall size scale of the experiment where very fast pressure-volumetemperature transformations occur along the free jet. Second, by reducing the collision frequency, since the collisional deficit effectively quenches the condensation process at some intermediate stage preventing the cluster size distribution to evolve forward into the condensed phase.

On the other hand, the volume $\mathscr{R}$ observed by Raman scattering has a section of $\approx 2 \mu \mathrm{m}$ along the flow axis, and the time $\Delta t$ for a cluster to cross the volume $\mathscr{R}$ is $\approx 2 \mathrm{~ns}$. Since the characteristic Raman scattering time is on the order of $10^{-13} \mathrm{~s}$, the clusters are perceived in the experiment as long-life stable species. The intensity of the Raman lines in the spectra on Fig. 1 (top) is the result of the accumulated spectral fingerprints of many clusters crossing volume $\mathscr{R}$ along the integration time of $60 \mathrm{~s}$ per multiplex recorded spectrum. Where necessary up to twenty spectra were accumulated in order to improve the signal to noise ratio. These intensities $I_{N}$ are proportional to the number of monomers in clusters of size $N$, leading to the fundamental quantity for the present work

$$
X_{N}=\frac{I_{N}}{\sum_{N} I_{N}}, \text { with } \sum_{N} X_{N}=1 .
$$


It represents the fractional abundance, $n_{N} / n$, of monomers in clusters of size $N,{ }^{16}$ and provides the essential information on the nucleation kinetics, as discussed below.

Fractions $X_{N}$ have been measured along a number of jets generated in the range of stagnation conditions $0.3 \leq P_{0} \leq 1.5$ bars and $45 \leq T_{0} \leq 90 \mathrm{~K}$. Combining this information with the $P T$-isentropic paths associated with the jets the time resolved non-equilibrium phase diagram of Fig. 2 (main) has been prepared. It describes semi-quantitatively the very first steps of condensation of $\mathrm{pH}_{2}$ in terms of pressure, temperature, and time, establishing the existence domains of the first clusters, in particular those of dimer and trimer. It should be noted that the same isentrope conveys the same clustering pattern independently of the stagnation conditions.

The jets studied here sample the upper region between CE and XD isentropes in Fig. 2. Several regions of maximum cluster size can be identified in this domain: to the right of the $\mathrm{CD}$ line only monomers and dimers were found, with no detectable amount of larger clusters. The CD boundary was determined by the presence or absence of the spectral line at $4160.358 \mathrm{~cm}^{-1}$, which is the fingerprint of the $\mathrm{pH}_{2}$ trimer. ${ }^{12}$ The monomer + dimer region is tentatively bounded at the low pressure end by the isentrope XD corresponding to $P_{0}=16$ bars and $T_{0}=295 \mathrm{~K}$, where no dimers were found. To the right of the XD isentrope no cluster was detected in spite of being inside the "solid" region of the phase diagram (note that $\mathrm{AXB}$ is the solid-gas boundary at equilibrium). Trimers were only found to the left of the $\mathrm{CD}$ line, with homologous boundaries $\mathrm{C}^{\prime} \mathrm{D}^{\prime}, \mathrm{C}^{\prime \prime} \mathrm{D}^{\prime \prime}$, etc., defining increasingly narrower regions for the sequential formation of larger clusters. The lower $P T$-end of the $\mathrm{CD}$ and $\mathrm{C}^{\prime} \mathrm{D}^{\prime}$ boundaries in Fig. 2 (dotted) is taken as a linear extrapolation, due to the lack of experimental data points. Actually, they could be bent due to the small amount of energy released by condensation and by the increasing collisional deficit along the expansion.

It must be stressed that the time domain for the existence of just dimers and monomers in the jets investigated here (Fig. 2) ranges between 100 and $300 \mathrm{~ns}$, depending on the particular isentrope. These times become progressively shorter for the onset of larger clusters, what is illustrative of the demanding experimental conditions for their systematic study. Present dimer abundances are comparable to those of static (bulk) $\mathrm{H}_{2}$ experiments, ${ }^{8}$ while the abundance of larger clusters is much higher in the present jets.

\section{Quantitative interpretation of condensation onset}

A major advantage of the present methodology is the sequential formation trend of the small clusters along the jet axis, where $X_{N}<X_{M}$ if $N>M$. So, by choosing judiciously the stagnation conditions $P_{0}$ and $T_{0}$, which control the collisional frequency, the growth process can be nearly "frozen" at a given size $N$. For instance, the evolution of $X_{N}$ fractions in two jets frozen at $N=2$ and $N=5$ is shown in Fig. 3. This enables truncating the nucleation kinetic equations at the desired cluster size, which is $N=3$ in this work.

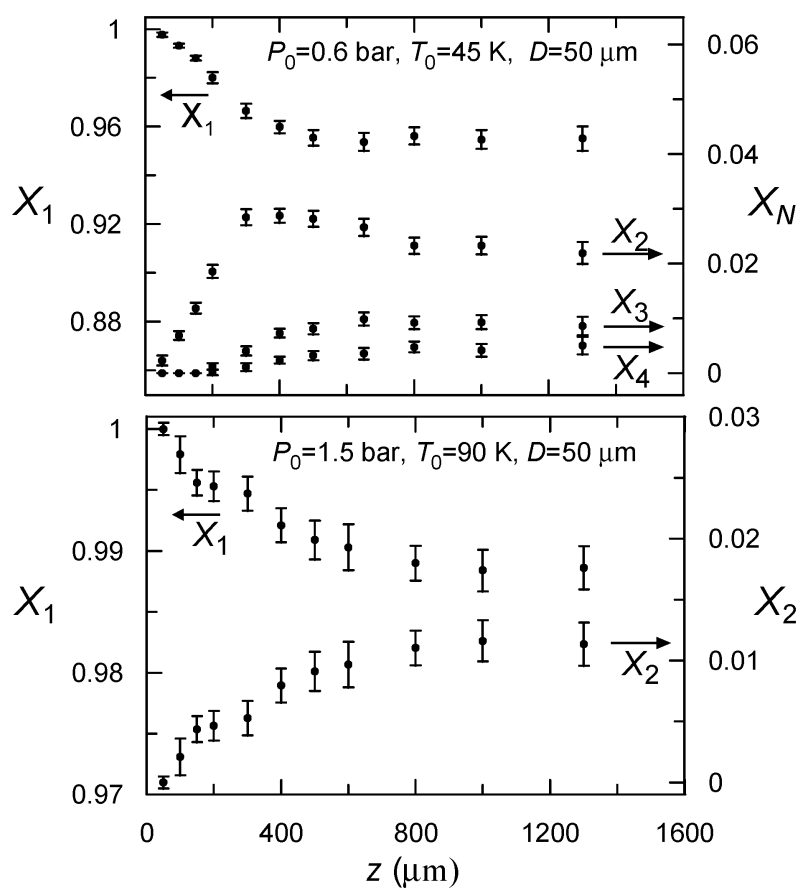

Fig. 3 Fractional abundances of monomers in clusters along two different supersonic jets frozen at $N=2$ (bottom) and $N=5$ (top). For clarity, $X_{5}<X_{4} / 2$ is not depicted.

In $\mathrm{pH}_{2}$ most molecules occupy at low temperature the ground rotational state $J=0$ and are like ${ }^{4} \mathrm{He}$ spinless bosons. The low mass of $\mathrm{H}_{2}$ and the weak $\mathrm{H}_{2}-\mathrm{H}_{2}$ intermolecular potential, although three to four times deeper than that of $\mathrm{He}-\mathrm{He}$ potential, suggests quantum delocalization and some similarities with ${ }^{4} \mathrm{He}$ in its collective physical properties.

While size distributions of $(\mathrm{He})_{N}$ clusters with $N>10$ have been explained on the basis of binary collisions, ${ }^{17}$ the formation of smaller clusters appears to be governed by threebody processes. ${ }^{1}$ By analogy between ${ }^{4} \mathrm{He}$ and $\mathrm{pH}_{2}$ at low temperature, and by the very few bound states of $\mathrm{pH}_{2}$ dimer ${ }^{18}$ and trimer, ${ }^{19}$ we consider the dimerization and trimerizacion of $\mathrm{pH}_{2}$ in the frame of the three-body processes

$$
\begin{gathered}
\mathrm{pH}_{2}+\mathrm{pH}_{2}+\mathrm{pH}_{2} \stackrel{k_{2}}{\longrightarrow}\left(\mathrm{pH}_{2}\right)_{2}+\mathrm{pH}_{2}, \\
\left(\mathrm{pH}_{2}\right)_{2}+\mathrm{pH}_{2}+\mathrm{pH}_{2} \stackrel{k_{3}}{\longrightarrow}\left(\mathrm{pH}_{2}\right)_{3}+\mathrm{pH}_{2},
\end{gathered}
$$

with net rates

$$
\begin{aligned}
& R_{2}=k_{2} n_{M}^{3}-k_{-2} n_{\mathrm{M}} n_{\mathrm{D}} / 2, \\
& R_{3}=k_{3} n_{M}^{2} n_{\mathrm{D}} / 2-k_{-3} n_{\mathrm{M}} n_{\mathrm{T}} / 3,
\end{aligned}
$$

where $n_{\mathrm{M}}, n_{\mathrm{D}}, n_{\mathrm{T}}$ are the number densities of free monomers, monomers in dimers, and monomers in trimers respectively; ${ }^{16}$ $k_{2}$ and $k_{3}$ are the dimer and trimer formation rate coefficients in processes (2) and (3), while $k_{-2}$ and $k_{-3}$ are their reverses. Since the growth model considered here is truncated at $N=3$, the $k_{3}$ trimer formation rate accounts for the dimer destruction rate within the approximation discussed below. The proposed three-body mechanism is consistent with that observed for $\mathrm{pH}_{2}$ dimer and trimer formation in the presence of $\mathrm{He}^{14}$ 


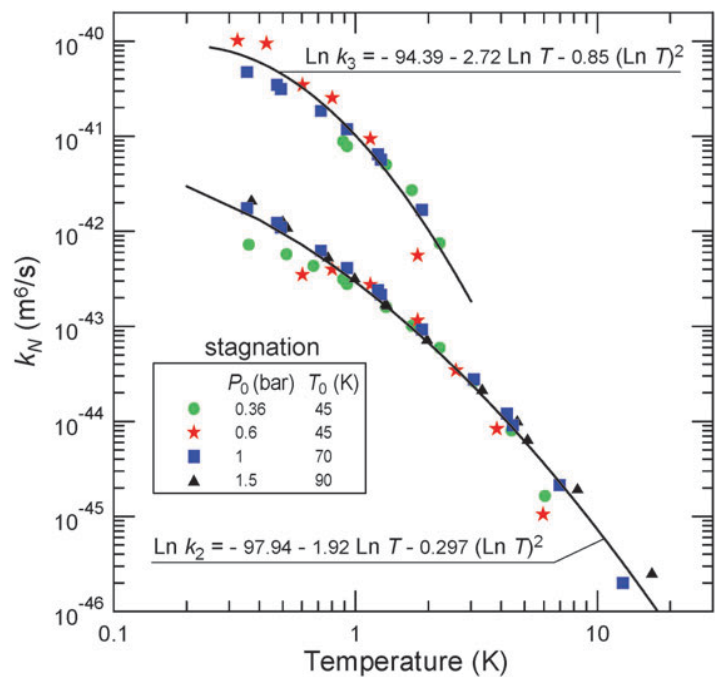

Fig. 4 Three-body $\mathrm{pH}_{2}$ dimer and trimer formation rates, $k_{2}$ and $k_{3}$, respectively.

The kinetic equations for the time evolution of $n_{\mathrm{M}}, n_{\mathrm{D}}$, and $n_{\mathrm{T}}$ along the jet are

$$
\begin{aligned}
& \mathrm{d} n_{\mathrm{M}} / \mathrm{d} t=-2 R_{2}-R_{3}-D_{\mathrm{g}} n_{\mathrm{M}}, \\
& \mathrm{d} n_{\mathrm{D}} / \mathrm{d} t=2 R_{2}-2 R_{3}-D_{\mathrm{g}} n_{\mathrm{D}}, \\
& \mathrm{d} n_{\mathrm{T}} / \mathrm{d} t=3 R_{3}-D_{\mathrm{g}} n_{\mathrm{T}},
\end{aligned}
$$

where $D_{\mathrm{g}}$ stands for the geometrical dilution factor along the expansion. ${ }^{1}$ The system (6) can be simplified in two ways. First, by employing the fractional abundances $X_{N}$ defined in (1) the terms in $D_{\mathrm{g}}$ in (6) vanish, as can be shown diferentiating the continuity equation along the jet. Second, neglecting the reverse processes in eqn (2) and (3), since the reaction quotient $n_{\mathrm{D}} / 2 n_{\mathrm{M}}^{2}$ along the jets always remains well below the statistical equilibrium constant at the local temperature. Actually, the observed dimers and trimers correspond to local PT-conditions within the "solid" region of the phase diagram of Fig. 2. The system (6) becomes reduced this way to the more operative form

$$
\begin{aligned}
& V(z) \mathrm{d} X_{1} / \mathrm{d} z=n^{2}(z)\left(-2 k_{2} X_{1}^{3}-(1 / 2) k_{3} X_{1}^{2} X_{2}\right), \\
& V(z) \mathrm{d} X_{2} / \mathrm{d} z=n^{2}(z)\left(2 k_{2} X_{1}^{3}-k_{3} X_{1}^{2} X_{2}\right), \\
& V(z) \mathrm{d} X_{3} / \mathrm{d} z=n^{2}(z)(3 / 2) k_{3} X_{1}^{2} X_{2},
\end{aligned}
$$

where $z, V(z), X_{N}(z), \mathrm{d} X_{N} / \mathrm{d} z, n(z)$ are experimental quantities, and only $k_{2}(z)$ and $k_{3}(z)$ remain as the unknowns to be determined. The latter are eventually transformed into $k_{2}(T)$ and $k_{3}(T)$ since the relation between position $z$ in the jet and temperature $T$ is known. The solutions to system (7) have been obtained along the four jets corresponding to the isentropic paths in the $P T$-diagram of Fig. 2. As mentioned above, different stagnation conditions on the same isentrope lead to the same clustering pattern, but at different time scales, producing redundant information (to experimental uncertainty) which confirms the robustness of the procedure. The threebody $\mathrm{pH}_{2}$ dimer and trimer formation rates obtained from system (7) are shown in Fig. 4. It should be noted that the trimer formation rate could not be established at $T \geq 2 \mathrm{~K}$, in agreement with Fig. 2.

Finally, it is worth comparing the formation rates for $\mathrm{pH}_{2}$ dimer and trimer with those for ${ }^{4} \mathrm{He}$ reported in ref. 1 . In both systems the same thermal trend is observed, with $k_{2}$ and $k_{3}$ decreasing for increasing temperature. The same $k_{N}$ rate for the two systems lay within two orders of magnitude for the thermal range investigated here. However, in this range $k_{3}$ is larger than $k_{2}$ for $\mathrm{pH}_{2}$, while this happens for ${ }^{4} \mathrm{He}$ only below $\approx 0.02 \mathrm{~K}^{1}$

\section{Conclusions and comments}

Two major conclusions are drawn from the present study. First, we demonstrate that the kinetics of the elementary processes involved in the onset of molecular condensation can be studied experimentally with unprecedented detail combining state-of-the-art Raman spectroscopy with cryogenic supersonic jets. Second, we find that the formation of nascent $\mathrm{pH}_{2}$ dimers and trimers is described accurately by three-body recombination processes whose rate coefficients $k_{2}$ and $k_{3}$ account for a large number of experimental data. Since $\left(\mathrm{pH}_{2}\right)_{N}$ clusters are a paradigm of condensing quantum systems, these rate coefficients provide a challenge to theory and to high-level calculations. ${ }^{3}$

Furthermore, by extending the cluster growth model, an improved mapping of the boundaries in Fig. 2 for $N \geq 3$, which converge fast to the liquid and solid as observed in ref. 12, may be conceived in the frame of the present experimental methodology. It may be conjectured that the predicted superfluid phase of $\left(\mathrm{pH}_{2}\right)_{N}$ clusters is confined between two such boundaries.

At any rate, the wealth of information described above in terms of a time dependent $P T$-phase diagram, cluster existence domains, fractional abundances, and rates accounting for a large number of experimental data, paves the way for a full understanding of the initial steps of molecular condensation.

\section{Acknowledgements}

This work was supported by the Spanish Ministerio de Ciencia e Innovación, projects FIS2004-02576 and FIS2007-61430. Thanks are due to J. P. Toennies and the Max-Planck-Institut für Strömungsforschung, Göttingen, for the loan of a $\mathrm{nH}_{2} \rightarrow \mathrm{pH}_{2}$ converter and a gas compressor.

\section{References}

1 L. W. Bruch, W. Schöllkopf and J. P. Toennies, J. Chem. Phys., $2002,117,1544$.

2 J. J. Breen, K. Kilgore, K. Stephan, R. Hofmann-Sievert, B. D. Kay, R. G. Keesee, T. D. Märk and A. W. Castleman, Jr., Chem. Phys., 1984, 91, 305.

3 R. T. Pack, R. B. Walker and B. K. Kendrick, J. Chem. Phys., 1998, 109, 6701; R. T. Pack, R. B. Walker and B. K. Kendrick, J. Chem. Phys., 1998, 109, 6714; J. W. Eerkens, Chem. Phys., 2001, 269, 189.

4 J. E. Cuervo and P.-N. Roy, J. Chem. Phys., 2008, 128, 224509; R. Guardiola and J. Navarro, J. Chem. Phys., 2008, 128, 144303; J. I. Martínez, M. Isla and J. A. Alonso, Eur. Phys. J. D, 2007, 43, 61; E. Rabani and J. Jortner, J. Phys. Chem. B, 2006, 110, 18893; 
S. Baroni and S. Moroni, ChemPhysChem, 2005, 6, 1884; G. E. López, J. Chem. Phys., 2002, 117, 2225.

5 P. Sindzingre, D. Ceperley and M. L. Klein, Phys. Rev. Lett., 1991 , 67, 1871; F. Mezzacapo and M. Boninsegni, Phys. Rev. Lett., 2008, 100, 145301; S. A. Khairallah, M. B. Sevryuk, D. M. Ceperley and J. P. Toennies, Phys. Rev. Lett., 2007, 98, 183401.

6 V. L. Ginzburg and A. A. Sobyanin, JEPT Lett., 1972, 15, 343; V. S. Vorob'ev and S. P. Malyshenko, J. Phys.: Condens. Matter, 2000, 12, 5071 .

7 S. Grebenev, B. Sartakov, J. P. Toennies and A. F. Vilesov, Science, 2000, 289, 1532; F. Paesani, R. E. Zillich, Y. Kwon and K. B. Whaley, J. Chem. Phys., 2005, 122, 181106.

8 A. R. W. McKellar, J. Chem. Phys., 1990, 92, 3261.

9 G. Tepper and D. Miller, Phys. Rev. Lett., 1992, 69, 2927.

10 A. van Deursen and J. Reuss, Int. J. Mass Spectr., 1973, 11, 483; E. L. Knuth, F. Schönemann and J. P. Toennies, J. Chem. Phys., 1995, 102, 6258; F. Buyvol-Kot, A. Kalinin, O. Kornilov, J. P. Toennies and J. A. Becker, Solid State Commun., 2005, 135, 532; O. Kornilov and J. P. Toennies, J. Chem. Phys., 2008, 128, 194306.
11 G. Chen, C. Wang, H. Lu, S. Li, J. Liu, G. Ni, R. Li and Z. Xu, J. Phys. B: At., Mol. Opt. Phys., 2007, 40, 445.

12 G. Tejeda, J. M. Fernández, S. Montero, D. Blume and J. P. Toennies, Phys. Rev. Lett., 2004, 92, 223401.

13 K. Kuyanov-Prozument and A. F. Vilesov, Phys. Rev. Lett., 2008 , 101, 205301.

14 S. Montero, J. H. Morilla, G. Tejeda and J. M. Fernández, Eur. Phys. J. D, 2009, 52, 31.

15 H. P. Godfried and I. F. Silvera, Phys. Rev. A: At., Mol., Opt. Phys., 1983, 27, 3019.

$16 n_{N}$ is the number density of $\mathrm{pH}_{2}$ monomers in clusters of size $N$, not to be confused withthe number density of clusters of size $N$, which is $n_{N} / N$. With our definition $n=\sum_{N} n_{N}$ is independent of cluster size distribution.

17 J. Chaiken, J. Goodisman, O. Kornilov and J. P. Toennies, J. Chem. Phys., 2006, 125, 074305.

18 R. J. Hinde, J. Chem. Phys., 2008, 128, 154308.

19 H.-G. Yu, J. Chem. Phys., 2004, 120, 2270; L. S. Costa and D. C. Clary, J. Chem. Phys., 2002, 117, 7512. 